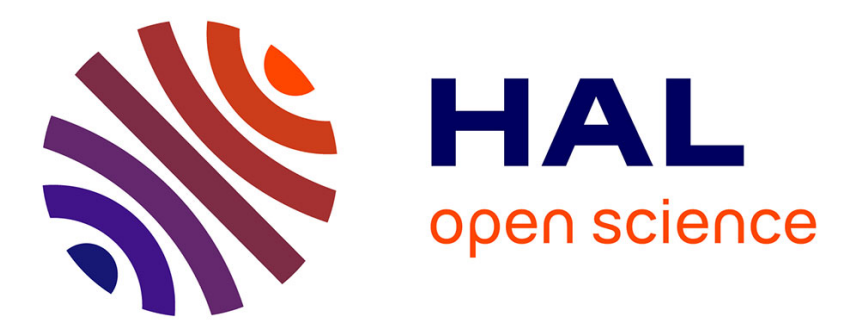

\title{
Grand Canonical Monte Carlo Simulation Study of Water Adsorption in Silicalite at $300 \mathrm{~K}$
}

\author{
Joël Puibasset, R.J.-M. Pellenq
}

\section{To cite this version:}

Joël Puibasset, R.J.-M. Pellenq. Grand Canonical Monte Carlo Simulation Study of Water Adsorption in Silicalite at 300 K. Journal of Physical Chemistry C, 2008, 112, pp.6390-6397. hal-00303812

\section{HAL Id: hal-00303812 \\ https://hal.science/hal-00303812}

Submitted on 9 Feb 2022

HAL is a multi-disciplinary open access archive for the deposit and dissemination of scientific research documents, whether they are published or not. The documents may come from teaching and research institutions in France or abroad, or from public or private research centers.
L'archive ouverte pluridisciplinaire HAL, est destinée au dépôt et à la diffusion de documents scientifiques de niveau recherche, publiés ou non, émanant des établissements d'enseignement et de recherche français ou étrangers, des laboratoires publics ou privés. 


\title{
Grand Canonical Monte Carlo simulation study of water adsorption in silicalite at $300 \mathrm{~K}$
}

\author{
Joël Puibasset ${ }^{1}$, Roland J.-M. Pellenq ${ }^{2}$ \\ ${ }^{1}$ Centre de Recherche sur la Matière Divisée, CNRS-Université d'Orléans, 1b, rue de la Ferollerie, \\ 45071 Orléans cedex 02, France \\ ${ }^{2}$ Centre de Recherche en Matière Condensée et Nanosciences, CNRS, Campus de Luminy, case 913, \\ 13288 Marseille cedex 09, France
}

puibasset@cnrs-orleans.fr 


\section{ABSTRACT}

This molecular simulation work focuses on the adsorption of water in a priori hydrophobic silicalite-1, a microporous ordered silica. The water-water interactions are described with the SPC model while water-silica interactions are calculated in the framework of the PN-TrAZ model. The water adsorption isotherm at $300 \mathrm{~K}$, the configurational energies, and the isosteric heat of adsorption are calculated by the Grand Canonical Monte Carlo (GCMC) simulation method. The thermodynamic integration scheme allows to calculate the grand potential along the adsorption isotherm. The adsorption results are compared with experiments, showing only qualitative agreement. Indeed, the simulations do not reproduce the expected hydrophobicity of silicalite (V. Eroshenko, R.-C. Regis, M. Soulard, J. Patarin, C. R. Phys. 2002, 3, 111). This indicates that common models used to describe confined polar molecules are far from being operative. In this work, it is shown, on the basis of periodic ab initio calculations, that confined water molecules in silicalite have a dipole value roughly 10 percent smaller than in the bulk liquid phase, indicating that the environment felt by a confined water molecule in silicalite pores is not equivalent to that in the bulk liquid. This suggests that effective intermolecular potentials parameterized for bulk water are inefficient to describe ultra confined water molecules. Reducing the SPC water dipole moment by $5 \%$ (i.e. decreasing water partial charges in magnitude) in GCMC calculations does allow reproducing the experimental water/silicalite isotherm at $300 \mathrm{~K}$.

KEYWORDS. Grand Canonical Monte Carlo, ab initio periodic calculations, water, silicalite, adsorption, intrusion 


\section{INTRODUCTION}

Many industrial processes like separation and catalysis involve Zeolites. ${ }^{1,2}$ From a fundamental point of view, zeolites are microporous models suitable to study fluid in extreme confinement. In such situation, fluid properties are expected to be drastically different from that of the bulk fluid or fluid confined in mesoporous materials. As a simple polar fluid, water constitutes a good candidate for studying adsorption properties in such microporous ordered systems. Industry is also interested in the development of hydrophobic zeolites for gas separation in the presence of water. From experimental observations, it is expected that dealuminated zeolites like silicalite be hydrophobic, despite the fact that the presence of defects strongly enhances their affinity for water. A recent application of such hydrophobic systems has also been proposed for storage or dissipation of mechanical energy, by intrusion/extrusion of water into/from the silicalite nanopores. ${ }^{3,4}$

Molecular simulations help to understand dynamical, structural or thermodynamic properties of confined water. ${ }^{5-12}$ The aim of this study is to calculate thermodynamic properties of confined water in silicalite with a molecular model which was successfully used to model water adsorption in disordered mesoporous silica substrate like Vycor glass. ${ }^{13-15}$ The adsorption isotherm, isosteric heat of adsorption, Helmholtz free energy, entropy and grand potential are calculated as a function of the chemical potential of water. The results show qualitative agreement with experimental data. However, water is shown to condense before saturating pressure. Two possible improvements of the model are proposed. The first one consists in decreasing the water/silicalite interactions by optimizing one repulsive parameter. The second one consists of reducing the permanent dipole of the water model, in order to take into account the fact that water adsorbed in silicalite is in a less polarizing media than in bulk water. 


\section{NUMERICAL DETAILS}

The atomistic numerical adsorbent is made of the $3 * 3 * 3$ repetition of one unit cell of the silicalite-1, as shown by the front view in Fig 1. The spatial dimensions of the simulation box are $60.21 * 59.76 * 40.26 \AA^{3}$. Periodic boundary conditions are applied in the 3 directions. The adsorbed water is described by the SPC model ${ }^{16}$, because it is a fast computable model which reproduces well the thermodynamic and structural properties of water around ambient temperature, like vapor pressure, enthalpy of vaporization, and radial distribution functions. ${ }^{17}$

The interaction of water with silicon and oxygen species is assumed to remain weak, in the physisorption energy range. In this work, we have used a TrAZ form of the original PN-type potential function as reported for adsorption of rare gases and nitrogen in silicalite- $1 .{ }^{18}$ The PN-TrAZ potential function is based on the usual partition of the adsorption intermolecular energy restricted to two body terms only: it includes a dispersion interaction term, a repulsive short range contribution and an induction term. The choice of this particular model to describe the water/adsorbent potential was motivated by the good degree of parameter transferability. Indeed, in a previous study ${ }^{13}$, we found that using a set of potential parameters previously derived for adsorption in silica zeolite augmented to take into account hydroxyl groups, the TrAZ model allows reproducing both low coverage experimental adsorption isotherm (amount adsorbed versus pressure at constant temperature) and isosteric heat curve with no further adjustment. In the TrAZ model, the adsorbate-surface energy $\left(u_{k \in A}^{i}\right)$ of the $\mathrm{k}^{\text {th }}$ atom of molecule $\mathrm{A}$ at a position $i$ in the simulation box, is given by

$u_{k \in A}^{i}=\sum_{j \in\{O, S i\}}\left[A_{k j} e^{-b_{k j} r_{i j}}-\sum_{n=3}^{5} f_{2 n} \frac{C_{2 n, k j}}{r_{i j}^{2 n}}\right]-\frac{1}{2} \alpha_{k} \boldsymbol{E}_{i}^{2}$

where $r_{i j}$ is the distance between the matrix species $\mathrm{j}$ and the position $i$ of the $\mathrm{k}^{\text {th }}$ atom of a given molecule, $f_{2 n}$ are so-called damping functions that depend on interspecies distance and on a repulsive 
parameter (see below) and finally $\boldsymbol{E}$ is the electric field created at adsorbate position $i$ due to the inorganic species ; all other symbols are physical parameters that depend on electronic properties of interacting species only. The sums runs over all $(j)$ atomic sites in the matrix that are silicon and oxygen atoms. The first term in the sum is a Born-Mayer term representing a two-body form of the short range repulsive energy due to finite compressibility of electron clouds when approaching the adsorbate at very short distance of the pore surface. There is one such a term per pair of interacting species. The repulsive parameters $\left(A_{k j}\right.$ and $\left.b_{k j}\right)$ are obtained from mixing rules of like-atoms pairs (see below). The second term in the above equation is a multipolar expansion series of the dispersion interaction in the spirit of the quantum mechanical perturbation theory applied to intermolecular forces. ${ }^{19}$ It has been shown that two (and three body) dispersion $C_{2 n}$ coefficients for isolated or in-condensed phase species can be obtained from the knowledge of the dipole polarizability and the effective number of polarizable electrons $N_{\text {eff }}$ of all interacting species ${ }^{18,20}$ which are closely related to partial charges that can be obtained from ab initio calculations. The $f_{2 n}$ terms in the above equation are damping functions of the form:

$$
f_{2 n}=1-\sum_{m=0}^{2 n}\left[\frac{\left(b^{k j} r_{i j}\right)^{m}}{m !}\right] e^{-b_{k j} r_{i j}}
$$

The role of these damping functions is to avoid divergence of the dispersion interaction at short distance where the wave functions of the two species overlap (i.e. when the interacting species are at contact). ${ }^{21}$ They allow taking into account the possible electronic exchange which has a non zero probability at short distance even for two close shell structures. For each pair of interacting species, they are parameterized with the single $b_{k j}$ repulsive parameter. The damped dispersion multipolar expansion can be seen as a convenient way to make the perturbation theory valid at short inter-atomic separations. The last term in equation (1) is the induction interaction as written in the context of the quantum mechanical perturbation theory applied to intermolecular forces. ${ }^{19}$ It represents an attractive energy arising from the coupling of the polarizable electronic cloud of the adsorbate atom of polarizability $\alpha_{k}$ at position $i$ with the electric field $E_{i}$ induced by the charges carried by framework species $(\mathrm{O}$ and $\mathrm{Si})$ that result from the bonding process within the inorganic matrix itself. In the case of water interacting with silicalite, one 
has to parameterize four different adsorbate/adsorbent-species potentials; all atomic parameters and coefficients are given in Table 1 and 2. Note that repulsive parameters for like-pairs are taken from a previous work on the simulation of rare gases in silicalite ${ }^{18}$ using Bohm and Ahlrichs ${ }^{22}$ combination rules based on $a b$ initio results at the Hartree-Fock level approximation which only describes the repulsive energy term in the case of non charged and polar species. This type of potential function based on the PN-TrAZ parameterization method was used in various studies of molecular and covalent fluids at interfaces from open surfaces ${ }^{13,23}$ to microporous zeolites ${ }^{24-28}$ and more recently in the case of mesoporous Vycor-like materials ${ }^{13-15,29-35}$.

Minimal image convention is adopted to calculate all interactions. Long range mean-field correction to dispersion terms cannot be precisely calculated because the density is not uniform. Actually, the size of the box is large enough so that large distance contributions are negligible. The electrostatic contribution is evaluated by summing on neutral subgroups of atoms of highest symmetry (tetrahedral silicon $\mathrm{SiO}_{4}$ ). Implementation of Ewald summation procedure $e^{36,37}$ has proven to be of little improvement, probably due to the large box size, absence of isolated charges.

For a given temperature, the Grand Canonical Monte Carlo (GCMC) technique ${ }^{38}$ allows to calculate the amount of water adsorbed in the silicalite sample in equilibrium with the above vapor at a given pressure. The repetition of the calculation for different vapor pressures enables to calculate the complete adsorption isotherm. As in experiments, to acquire the adsorption isotherm, an equilibrated configuration is used as an initial configuration for the next pressure. An equal number of trial for translation, rotation, and creation or destruction of molecules has been chosen for each Monte Carlo (MC) step at fixed chemical potential. The configurations are considered for visual examination and statistical calculations every block of $10^{4}$ Monte Carlo steps $\left(4.10^{4} \mathrm{MC}\right.$ trials) along the Markov chain, so that they are de-correlated. The Fig 2 shows a typical equilibration run at a relative pressure of 0.34 from an initial configuration at relative pressure 0.23 . The number of particles is drawn as a function of 
the number of blocks of $10^{4} \mathrm{MC}$ steps. As can be seen, equilibration is reached after approximately $2 \times 10^{4}$ blocks, that is to say around $3 \times 10^{5}$ trials per water molecule. To acquire data, another $5 \times 10^{5}$ trials per molecule were run, where the number of particles fluctuates around a constant value. After the complete saturation of the silicalite sample is reached for high chemical potential $\mu$, one can start decreasing $\mu$ to acquire the desorption branch (hysteresis).

\section{ADSORPTION ISOTHERMS AND CONFIGURATIONAL ENERGIES}

The figure 3 shows the adsorption/desorption isotherm obtained at $300 \mathrm{~K}$ as a function of the chemical potential or relative pressure $\mathrm{P} / \mathrm{P}_{0}$ where $\mathrm{P}_{0}=0.044$ bar is the saturating vapor pressure of $\mathrm{SPC}$ water at $300 \mathrm{~K}^{39,40}$ As can be seen the curve is reversible, as expected for a microporous adsorbent. The curve shows low initial adsorption for $\mathrm{P} / \mathrm{P}_{0}<0.1$, as for a hydrophobic surface. This is in qualitative agreement with experimental data. However, the substrate saturates for pressure lower than the saturating vapor pressure, which indicates a noticeable affinity of our numerical substrate for water. This point seems in disagreement with experimental data, which show stronger hydrophobicity. ${ }^{41-44}$ The energetic quantities calculated in the system confirm this point. This excessive hydrophilic character was previously observed by other authors reporting simulation results for confined water in silicalite with the same kind of approach based on a bulk water potential with a physisorption water-host potential. ${ }^{11}$ Several ways for model improvement are considered in the last part of the paper. In all cases, improvement of the model results in a shift of the adsorption properties towards higher chemical potential (more hydrophobic), in quantitative agreement with experimental data. However, the shape of the adsorption isotherm is not affected. This is why the thermodynamic analysis is performed with the non-optimized simulation results, the corrections then consisting in a simple shift in chemical potential. 
Figure 4 shows molecular configurations of water adsorbed in the numerical sample for different pressures. For clarity, the substrate is not shown. For low pressure $\left(\mathrm{P} / \mathrm{P}_{0}=0.0886\right)$ the system is made of isolated water molecules and clusters containing few molecules adsorbed on the substrate. For a relative pressure 1.67 times larger $\left(\mathrm{P} / \mathrm{P}_{0}=0.1477\right)$ the amount of water adsorbed is 4.5 times larger, and essentially all molecules are implied in a cluster (no isolated molecules left). At $\mathrm{P} / \mathrm{P}_{0}=0.2045$, the system is almost saturated, and the clusters merge into large size ones. The last snapshot shows the $\mathrm{P} / \mathrm{P}_{0}$ $=0.341$ configuration almost completely filled, with few adsorption sites left.

The Figure 5 gives the molecular configurational energy (in $\mathrm{kJ}$ per mol of water molecules) at $300 \mathrm{~K}$ as a function of the relative pressure (upper figure) or coverage (lower figure). The general trend shows that the configurational energy decreases with pressure or coverage: the water-silicalite interactions are then weaker than water-water interactions, which is characteristic of a hydrophobic adsorbent. More precisely, the evolution of the configurational energy with coverage shows essentially two behaviors. For relative pressure lower than 0.2 the energy decreases rapidly with relative pressure, whereas above $\mathrm{P} / \mathrm{P}_{0}=0.2$ the configurational energy hardly decreases with pressure. The first range of pressure corresponds to the initial adsorption of molecules on small and disconnected clusters previously adsorbed (see Fig. 4). On the other hand, for larger pressure essentially only one (infinite) cluster persists, and the number of free sites decreases significantly. These differences in the organization of the fluid explain the values of the configurational energy: at low pressure, the fragmentation of the fluid in small clusters, favored by entropy considerations (to be calculated later), reduces water-water interactions. The higher the pressure, the lager the clusters, and the stronger the fluid-fluid interactions, which decreases the configurational energy. Above a relative pressure of 0.2 , the system is almost completely filled. This saturation induces a change of slope of configurational energy versus relative pressure. The second graph, giving the configurational energy as a function of the coverage, shows a different behavior. The knee previously observed for relative pressure $\mathrm{P} / \mathrm{P}_{0}=0.2$ is not visible any more for the corresponding coverage of $2.57 \mathrm{mmol} / \mathrm{g}$. This confirms the previous analysis suggesting that the 
knee in configurational energy versus relative pressure is reminiscent of the knee in adsorption, which separates the rapid adsorption regime for $\mathrm{P} / \mathrm{P}_{0}<0.2$ from saturation regime for $\mathrm{P} / \mathrm{P}_{0}>0.2$. However, three portions can be seen. In the first and last part, for low coverage (less than $0.5 \mathrm{mmol} / \mathrm{g}$ ) and high coverage (larger than $4 \mathrm{mmol} / \mathrm{g}$ ) one observes a strong decrease of the energy with coverage, while for intermediate coverage values, the energy decrease is less important. These observations can be interpreted as follows: as previously, for intermediate coverage the configurational energy decreases with increasing coverage because the water-water interactions are comparable to water-silicalite interactions. The adsorption consists essentially in growth and coalescence of water clusters, which establish more hydrogen bonds, and improve the energetic cohesiveness of the system. For large coverage (more than $4 \mathrm{mmol} / \mathrm{g}$ ) the energetic gain is higher because the new molecules insert in sites where they can establish two hydrogen bonds. Such sites can be seen in Fig 4 for $\mathrm{P} / \mathrm{P}_{0}=0.341$, corresponding to "missing molecules" within the network. The addition of molecules in such sites result in an enhanced increase in cohesiveness of the fluid. The second feature to be explained takes place around $0.5 \mathrm{mmol} / \mathrm{g}$. Below this value, the configurational energy per molecule decreases slightly faster than above. In parallel, inspection shows that this crossover coverage also corresponds to the point where isolated molecules disappear at the expense of larger clusters (two or more than two molecules). The smaller the cluster, the larger the relative gain when adding a new molecule. In effect, whatever the size of an isolated cluster, the energetic gain per molecule when adding a new molecule is one hydrogen bond, divided by the size of the cluster. This explains why the lower the coverage, the steeper the slope of the configurational energy versus coverage, especially when one-molecule-size clusters are abundant.

The configurational energy is not a directly accessible quantity, from an experimental point of view. The measurable quantity is the isosteric heat of adsorption, which measures the heat released by the adsorption of a given amount of fluid, as a function of the total amount of fluid previously adsorbed in the substrate. Numerically, this quantity is deduced from cross-fluctuations in energy (U) and adsorbed quantity $(\mathrm{N})$ through the formula: ${ }^{38}$ 
$\mathrm{q}_{\mathrm{st}}=-\frac{\langle\mathrm{UN}\rangle-\langle\mathrm{U}\rangle\langle\mathrm{N}\rangle}{\left\langle\mathrm{N}^{2}\right\rangle-\langle\mathrm{N}\rangle^{2}}+\mathrm{RT}$

The result is given in Fig. 6. The general trend is an increase of the isosteric heat of adsorption with coverage, typical of hydrophobic adsorbent. The low coverage value gives information on the strength of the adsorbate/adsorbent interaction. At $300 \mathrm{~K}$, the heat released in the first steps of adsorption reaches $43 \mathrm{~kJ} / \mathrm{mol}$, a value close to the latent heat of vaporization of water. The water/silicalite interactions are then comparable in intensity to the water/water interactions, which is drastically different from hydrophilic substrate like silica surfaces presenting hydroxyl groups ${ }^{13,33}$, for which the water/silica interactions are much stronger than water/water interactions. However, the higher the coverage the higher the heat released. The contribution from the substrate (around $40 \mathrm{~kJ} / \mathrm{mol}$ ) is almost constant, due to the small size of the pores, while the contribution from water/water interactions increases (up to $10 \mathrm{~kJ} / \mathrm{mol}$ ) with the pore filling up. However, the water/water contribution can never reach its bulk value because the structure of water in silicalite is strongly distorted. Inspection shows that, at saturation, most of the molecules establish two hydrogen bonds instead of four in bulk water, which explains the increase of $10 \mathrm{~kJ} / \mathrm{mol}$ in isosteric heat.

\section{GRAND POTENTIAL, HELMHOLTZ FREE ENERGY AND ENTROPY CALCULATION}

The system is completely characterized from a thermodynamic point of view if one calculates the thermodynamic potential in grand canonical ensemble, the grand potential $\Omega=E-T S-\mu N$, where $E$ is the total internal energy, $T$ the temperature, $S$ the entropy, $\mu$ the chemical potential, and $N$ the number of particles in the system. This quantity is not a direct output of the GCMC simulation, but is easily calculated from the differential equation: ${ }^{45}$ 
$\left(\frac{\partial \Omega}{\partial \mu}\right)_{T, V}=-N$

where $N$ is the number of particles in the simulation box of volume $V$. To fix the absolute value for $\Omega$ one has to consider the low chemical potential region, where the adsorption isotherm follows essentially the ideal gas approximation ( $\mathrm{N}$ is proportional to the activity $e^{\beta \mu} / \Lambda^{3}$ ). In this case, the integration of equation 4 from minus infinity up to the first chemical potential values calculated by GCMC gives:

$\Omega_{i d}=-N k_{B} T$

where $k_{B}$ is Boltzmann's constant. The result for the grand potential obtained along the isotherm is shown in Fig. 7. It is normalized to the volume of the substrate, and given in $\mathrm{J} / \mathrm{cm}^{3}$ or MPa. For comparison the same quantity is also reported for bulk water at $300 \mathrm{~K}$. Is the bulk case, the system being homogeneous, the grand potential divided by the volume is equal to minus the pressure of the fluid. The confinement obviously strongly affects the thermodynamic potential. In the low chemical potential region, the grand potential is much lower in silicalite- 1 than in bulk water, because the density is much higher in the adsorbent. For large chemical potential values, both grand potential follow straight lines, due to the incompressibility of the dense phase. The slope is given by the average density of the fluid over the volume of the system, which is much lower for water adsorbed in silicalite due to the fraction occupied by the substrate. Since the definition of accessible volume for water molecules in microporous adsorbent is quite arbitrary, it is not possible to compare precisely the slopes, which should however be in a ratio close to the porosity of the substrate. The second point to note is the absence of discontinuity in the grand potential: the liquid-gas phase transition disappears in microporous adsorbent, as expected. This is reminiscent of what is observed for supercritical fluids. ${ }^{46}$

The Fig. 8 gives the Helmholtz free energy per particle

$f=\frac{F}{N}=\frac{\Omega}{N}+\mu$

and plotted as a function of the amount of adsorbed water. The ideal rotational contribution to molecular free energy $-11.1 \mathrm{~kJ} / \mathrm{mol}$ is not taken into account. As observed, the free energy increases continuously 
from low values (due to the large entropy of diluted phase) up to liquid-like values. For comparison, the bulk water free energy is equal to $-45.6 \mathrm{~kJ} / \mathrm{mol}$ in the liquid phase above the saturating pressure. In silicalite, the free energy is slightly lower than in the dense bulk phase due to interactions with the substrate: indeed, the entropy is lower than in bulk water, as discussed now.

The entropy of the system is conceptually very important since it measures the number of accessible states for the system, and then the degree of confinement. However, this quantity is rarely directly calculable, except by means of very sophisticated methods. Nevertheless, it can be deduced from the molecular Helmholtz free energy $f$ and the molecular configurational energy $u$, according to the formula: $s=\frac{3}{2} R+\frac{u-f}{T}$

where $R$ is the ideal gas constant $\left(8.314 \mathrm{JK}^{-1} \mathrm{~mol}^{-1}\right)$, arising from the kinetic part of the internal energy of water molecules. Here again, the rotational ideal gas contribution $49.5 \mathrm{JK}^{-1} \mathrm{~mol}^{-1}$ is omitted. The Fig. 9 shows the entropy as a function of the chemical potential $\mu$. The values are always smaller than the bulk water values: in the gas region, which extends from $\mu=-52$ up to $-45.6 \mathrm{~kJ} / \mathrm{mol}$ the entropy ranges from 194 down to $174 \mathrm{JK}^{-1} \mathrm{~mol}^{-1}$, whereas in the liquid region the entropy is almost constant and equals to 26.47 $\mathrm{JK}^{-1} \mathrm{~mol}^{-1}$ which is again larger than for water confined in silicalite. The reduction in entropy per molecule is a direct consequence of the loss in translation and rotation mobility due to the confinement in silicalite. This result, although expected, was never shown previously by simulation calculations for water confined in microporous system.

\section{IMPROVEMENT OF THE MODEL}

Let us now focus on a quantitative comparison of simulation results with experiments. The experimental data found in the literature are reported in Fig. 10. Various techniques are used: gravimetric or calorimetric measurements. ${ }^{41-44}$ In each case, the water uptake is very low, showing that 
silicalite is hydrophobic. Inspection of experimental data show large variations, probably due to differences in sample preparation. Above the bulk saturating point, conventional techniques fail. Eroshenko et al. ${ }^{3,4}$ developed intrusion experiments to measure the amount of water introduced in the porous material as a function of the liquid water pressure. The results show a linear volume variation of the system with pressure, with a rapid water uptake around $100 \mathrm{MPa}$. Two water intrusion points from the above mentioned experiments are reported in Fig. 10 , corresponding to $\mathrm{P} / \mathrm{P}_{0}=0.5$ and $\mathrm{P}=100 \mathrm{MPa}$. The water pressures have been converted to chemical potentials using the fact that the gas state is almost ideal, and by integrating the water equation of state above the saturating point, according to the standard procedure. As previously mentioned, the simulation results are in qualitative agreement with experimental results. However, the adsorption isotherm, as predicted by the model, is shifted towards low chemical potential as compared to experimental data: the pore filling occurs around $\mathrm{P} / \mathrm{P}_{0}=0.2$ in simulation $(\mu=-49 \mathrm{~kJ} / \mathrm{mol})$, whereas it is expected to occur around $\mu=-44 \mathrm{~kJ} / \mathrm{mol}$. Similar results where obtained by Desbiens et al. ${ }^{11}$ when using the same electrostatic partial charges for silicalite species. In order to reconcile simulation results with experimental data, two routes were considered to improve the model. The aim is essentially to reduce water/silicalite interactions to shift the adsorption to higher chemical potential. The nature of the interactions is twofold: dispersion/repulsion terms and electrostatic terms, giving the two possible routes. The first way consists in increasing one of the repulsion parameters of the PN-TrAZ model. More specifically, since most of the energetic contributions rise from the oxygen - oxygen interactions between water molecules and silica, the

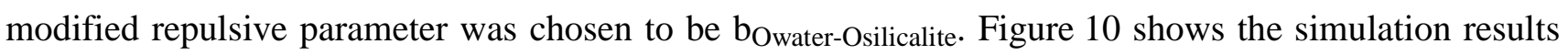
for a value of this parameter equal to $97 \%$ of its original value (reducing b increases the repulsion strength). As can be seen, a quantitative agreement with experimental data is obtained. This suggests that the initial model underestimates the water-silica repulsive term. This proposed re-parameterization offers a simple procedure to perform quantitative simulations of water adsorption in silicalite. Let us now consider the second possible route which consists in re-considering the electrostatic contributions. Desbiens et al. ${ }^{11}$ proposed to modify the partial charges of the silicalite species $\left(\mathrm{q}_{\mathrm{Si}}=1.4\right.$ instead of 2.0). 
Another way to modify electrostatic contributions is guided by the fact that the water model used in the simulation (SPC) might not be optimal to describe low density water. As a matter of fact, it is expected that the partial charges on a water molecule are greatly influenced by the environment seen by this molecule. In most cases, water simulation models are parameterized in the liquid state. In such a situation, the water molecules are surrounded by a highly polarizing media. It is then questionable whether such models are efficient to quantitatively describe the water clusters observed in silicalite. It is then suggested to consider the influence of water partial charges on water adsorption isotherm in silicalite.

To this end, we performed full ab initio Hartree-Fock calculations of water in the liquid bulk phase and confined to silicalite pores using the CRYSTAL98 code that allows periodic calculations. The choice of atomic basis sets is of central importance for such calculations. We have chosen 6-31G* orbital sets for hydrogen and oxygen species; silicon was modeled using a Durand pseudo-potential (http://www.crystal.unito.it/Basis_Sets/Ptable.html). This type of calculations allows obtaining (Mulliken) partial charges of all atoms in the considered periodic simulation box, hence characterizing the nature of bonding processes (ionic, covalent, ioni-covalent). Note however that these calculations were carried out at the Hartree Fock level of approximation thus ignoring electronic correlation effects hence dispersion interactions. As expected from Pauling's rule for electronegativity, we found that the partial charges of zeolitic species are half their formal values $\left(\mathrm{q}_{\mathrm{oZ}}=-1.05 \mathrm{e}, \mathrm{q}_{\mathrm{Si}}=+2.1 \mathrm{e}\right)$ in agreement with previous ab initio calculations on quartz. ${ }^{47}$ As shown in Figure 11 with such relatively large basis sets, a single water molecule having the SPC geometry $\left(\mathrm{HOH}=109.45^{\circ}, \mathrm{OH}=1 \AA\right)$ has a dipole moment norm at $1.67 \mathrm{D}(9 \%$ smaller than the experimental value, $1.85 \mathrm{D}$; note that the SPC value is fixed at 2.245 D). Such a discrepancy indicates the difficulty in calculating ab initio such an electronic property that is indeed very dependent on the basis sets; ${ }^{48}$ for instance the use of minimal STO3G basis sets leads to a dipole moment norm of $0.7 \mathrm{D}$ for the SPC water molecule geometry. Note that the recent work of Dyer and Cummings ${ }^{49}$ shows that DFT-CPMD (Car-Parinello ab initio molecular dynamics) 
calculations of the dipole moment distribution for water bulk liquid is also very dependent on the analysis technique: electron density partitioning among molecules following the Bader approach to determine partial charges leads to a dipole moment mean value of $2.5 \mathrm{D}$ at $300 \mathrm{~K}$ rather close to the experimental value, while the use of localized Wannier functions leads to dipole moment mean value of 3.1 D.

In this work, we first consider a molecular configuration of bulk water at $300 \mathrm{~K}$ (density $1 \mathrm{~g} / \mathrm{cc}$ ) generated from a Gibbs ensemble simulation. The ab initio distribution of dipole (see Fig 11) is centered on a mean value of 2.0 D (again smaller than the experimental value of 2.6 D). Let us now consider a confined phase of 27 water molecules in a unit cell of silicalite (loading relatively close to maximum). The calculation requires a very large hard disk temporary storage capacity ( 200 GB : number of atoms in the simulation cell $=363$ i.e. 4152 atomic orbitals and 2160 electrons). We found that the water dipole moment distribution is down-shifted to a mean value at $1.83 \mathrm{D}$, i.e. $9.5 \%$ smaller than the bulk value. This is an interesting result that shows that the electric field felt by a given water molecule due to the zeolite species and neighboring water molecules is not as intense as in the bulk. The same result was recently observed by Coudert et al ${ }^{50}$ performing DFT-CPMD calculations of water molecule confined to LTA zeolite. In addition, they show that the dipole moment shift is loading-dependent. Note that our ab initio results confirm the elegant results of Smirnov and Bougeard ${ }^{51}$ who performed classical molecular dynamics with charge-adaptative potentials of water confined in silicalite. Our approach captures correctly the underlying physics, and clearly shows the importance of polarization effects. It is however mentioned that to be fully self-consistent, it is preferable to perform classical simulations of confined polar fluid with polarizable potentials. The main physical conclusion is this work is that the reduction of the dipole moment of confined water molecules in siliceous zeolite (such as silicalite) is at the origin of the hydrophobic behavior of these materials. One may also infer that aluminum-containing zeolites have a hydrophilic character that is due to the strong electric field created by counterions in the zeolitic voids in direct contact with the water adsorbed phase. ${ }^{52}$ 
Figure 10 shows the simulation results obtained with partial charges on water molecules equal to $95 \%$ of the initial SPC model (5\% reduction of the water dipole moment). As can be seen, quantitative agreement with the experimental data is obtained, i.e. the pore filling occurs around a chemical potential $\mu=-44 \mathrm{~kJ} / \mathrm{mol}$. A more accurate description would take into account the molecule environment, and make the partial charges and hence the dipole moment, dependent on it "on the flight" as reported in Smirnov and Bougeard paper. ${ }^{51}$

\section{CONCLUSION}

The adsorption of water in ordered microporous silicalite- 1 has been analyzed by GCMC simulation method at $300 \mathrm{~K}$. The calculations were performed in the framework of the PN-TrAZ model, giving all interaction parameters between water species and silica substrate. The adsorption isotherm at $300 \mathrm{~K}$ is of type $\mathrm{V}$ without hysteresis, in qualitative agreement with experimental data. The isosteric heat of adsorption is also calculated. Its value is shown to increase as a function of water coverage. This is expected in a system which does not present a strong surface affinity with water molecules, as silicalite. The thermodynamic integration procedure is used to compute the Helmholtz free energy and grand potential, as well as molecular entropy which is shown to decrease with water coverage. Visual inspection of molecular configurations shows that water molecules form aggregates of larger and larger size as the chemical potential increases. In order to improve the quantitative agreement with experimental data, two modifications have been proposed. The first one consists in fine tuning the repulsive parameter between oxygen species to calculate the water/silicalite interactions. Reducing the repulsive parameter $\mathrm{b}_{\text {Owater-Osilicalite }}$ to $97.75 \%$ of its initial value given by the PN-TrAZ model allows a quantitative agreement with experimental data. This suggests that the model slightly underestimates the 
electronic repulsion between water and silicalite oxygen species. The second possibility to improve the agreement between GCMC and experimental data is to decrease the partial charges of the water model (hence its dipole moment). This is justified by the fact that the water models are generally parameterized for the bulk liquid phase, taking phenomenologically into account the polarization contributions. These contributions are expected to be lower in silicalite (lower electric fields due to tetrahedral silicon instead of dipolar contributions in bulk liquid water) as shown by quantum calculations of the effective partial charges of water molecules confined in silicalite (molecular configurations taken from the GCMC runs with the SPC model). These calculations suggest that the dipole of water confined in silicalite is roughly 10 percent lower than its bulk value. GCMC calculations performed with a SPC model with reduction of the partial charges by $5 \%$ allow a quantitative agreement with experimental data that indeed show a strong hydrophobic character. Further quantum chemical calculations are underway to evaluate the change in the water moment in aluminum-containing zeolites and find the reason of the highhydrophillicity at a molecular level.

\section{ACKNOWLEDGMENTS}

Drs N. Desbiens, A Boutin and I. Demachy are acknowledged for fruitful discussions. The simulations were performed on workstations purchased thanks to grants from Région Centre (France), and on IBM SP4 supercomputers at the Centre de Ressources Informatique de Haute Normandie (CRIHAN, St Etienne du Rouvray, France) and the Institut de Développement des Ressources en Informatique Scientifique (IDRIS-CNRS, Orsay, France). 


\section{REFERENCES}

(1) Handbook of Zeolite Science and Technology; Auerbach, S. M.; Carrado, K. A.; Dutta, P. K., Eds.; Marcel Dekker: New York, 2003.

(2) Patarin, J.; Gies, H. Crystalline and organized porous solids (thematic issue). In C. R. Chim., 2005; Vol. 8.

(3) Eroshenko, V.; Regis, R.-C.; Soulard, M.; Patarin, J. J. Am. Chem. Soc. 2001, 123, 8129.

(4) Eroshenko, V.; Regis, R.-C.; Soulard, M.; Patarin, J. C. R. Physique 2002, 3, 111.

(5) Bussai, C.; Hannongbua, S.; Haberlandt, R. J. Phys. Chem. B 2001, 105, 3409.

(6) Bussai, C.; Vasenkov, S.; Liu, H.; Böhlmann, W.; Fritzsche, S.; Hannongbua, S.;

Haberlandt, R.; Kärger, J. Appl. Catal. A: Gen. 2002, 232, 59.

(7) Bussai, C.; Fritzsche, S.; Haberlandt, R.; Hannongbua, S. J. Phys. Chem. B 2003, 107, 12444.

(8) Demontis, P.; Stara, G.; Suffritti, G. B. J. Phys. Chem. B 2003, 107, 4426.

(9) Fleys, M.; Thompson, R. W. J. Chem. Theory Comput. 2005, 1, 453.

(10) Fleys, M.; Thompson, R. W.; MacDonald, J. C. J. Phys. Chem. B 2004, 108, 12197.

(11) Desbiens, N.; Boutin, A.; Demachy, I. J. Phys. Chem. B 2005, 109, 24071.

(12) Ramachandran, C. E.; Chempath, S.; Broadbelt, L. J.; Snurr, R. Q. Micropor. Mesopor. Mater. 2006, 90, 293.

(13) Puibasset, J.; Pellenq, R. J.-M. J. Chem. Phys. 2003, 118, 5613.

(14) Puibasset, J.; Pellenq, R. J.-M. J. Chem. Phys. 2003, 119, 9226.

(15) Puibasset, J.; Pellenq, R. J.-M. J. Chem. Phys. 2005, 122, 094704.

(16) Berendsen, H. J. C.; Postma, J. P. M.; van Gunsteren, W. F.; Hermans, J. In Intermolecular Forces; B. Pullman: Dordrecht: Reidel, 1981; pp 331. 
(17) Jorgensen, W. L.; Chandrasekhar, J.; Madura, J. D.; Impey, R. W.; Klein, M. L. J. Chem. Phys. 1983, 79, 926.

(18) Pellenq, R. J.-M.; Nicholson, D. J. Phys. Chem. 1994, 98, 13339.

(19) Stone, A. The Theory of Intermolecular ForcesOxford: Clarendon, 1996.

(20) Pellenq, R. J.-M.; Nicholson, D. Mol. Phys. 1998, 95, 549.

(21) Tang, K. T.; Toennies, J. P. J. Chem. Phys. 1984, 80, 3726.

(22) Bohm, H. J.; Ahlrichs, R. J. Chem. Phys. 1982, 77, 2028.

(23) Marinelli, F.; Grillet, Y.; Pellenq, R. J.-M. Mol. Phys. 1999, 97, 1207.

(24) Nicholson, D.; Pellenq, R. J.-M. Adv. Colloid Interface Sci. 1998, 76/77, 179.

(25) Fuchs, A. H.; Cheetham, A. K. J. Phys. Chem. B 2001, 105, 7375.

(26) Grey, T. J.; Nicholson, D.; Gale, J. D.; Peterson, B. K. Appl. Surf. Sci. 2002, 196, 105.

(27) Bichara, C.; Yves-Raty, J.; Pellenq, R. J.-M. Phys. Rev. Lett. 2002, 89, 016101.

(28) Lachet, V.; Boutin, A.; Tavitian, B.; Fuchs, A. H. J. Phys. Chem. B 1998, 102, 9224.

(29) Pellenq, R. J.-M.; Rousseau, B.; Levitz, P. E. Phys. Chem. Chem. Phys. 2001, 3, 1207.

(30) Pellenq, R. J.-M.; Levitz, P. E. Mol. Phys. 2002, 100, 2059.

(31) Coasne, B.; Pellenq, R. J.-M. J. Chem. Phys. 2004, 120, 2913.

(32) Puibasset, J.; Pellenq, R. J.-M. 6th Int. Symp. on the Characterization of Porous Solids, in Studies in Surface Science and Catalysis, Vol 144, p371, Alicante, Spain, F. Rodriguez Reinoso, B. McEnaney, J. Rouquerol and K. Unger 2002.

(33) Puibasset, J.; Pellenq, R. J.-M. The European Physical Journal E 2003, 12, S67.

(34) Puibasset, J.; Pellenq, R. J.-M. J. Phys.: Condens. Matter 2004, 16, S5329.

(35) Puibasset, J.; Pellenq, R. J.-M. Eur. Phys. J. 2007, ST 141, 41.

(36) Ewald, P. Annalen der Physik 1921, 64, 253.

(37) Heyes, D. M. Phys. Rev. B 1994, 49, 755.

(38) Nicholson, D.; Parsonage, N. G. Computer Simulation and the Statistical Mechanics of Adsorption; Academic Press: London, 1982. 
(39) Errington, J. R.; Panagiotopoulos, A. Z. J. Phys. Chem. B 1998, 102, 7470.

(40) Vorholz, J.; Harismiadis, V. I.; Rumpf, B.; Panagiotopoulos, A. Z.; Maurer, G. Fluid Phase Equilibria 2000, 170, 203.

(41) Giaya, A.; Thompson, R. W. Micropor. Mesopor. Mater. 2002, 55, 265.

(42) Fubini, B.; Bolis, V.; Bailes, M.; Stone, F. S. Solid State Ionics 1989, 32/33, 258.

(43) Zhao, X. S.; Ma, Q.; Lu, G. Q. Energy and Fuels 1997, 12, 1051.

(44) Kenny, M. B.; Sing, K. S. W. Chem. Ind. (London) 1990, 2, 39.

(45) Peterson, B. K.; Gubbins, K. E. Molecular Physics 1987, 62, 215.

(46) Puibasset, J. J. Phys. Chem. B 2005, 109, 480.

(47) Nada, R.; Catlow, C. R. A.; Dovesi, R.; Pisani, C. Phys. Chem. Minerals 1990, 17, 353.

(48) Martin, F.; Zipse, H. J. Comp. Chem. 2005, 26, 97.

(49) Dyer, P. J.; Cummings, P. T. J. Chem. Phys. 2006, 125, 144519.

(50) Coudert, F. X.; Vuilleumier, R.; Boutin, A. Chem. Phys. Chem. 2006, 7, 2464.

(51) Smirnov, K. S.; Bougeard, D. Chem. Phys. 2003, 292, 53.

(52) Di Lella, A.; Desbiens, N.; Boutin, A.; Demachy, I.; Ungerer, P.; Bellat, J.-P.; Fuchs, A. H. Physical Chemistry Chemical Physics 2006, 8, 5396. 
FIGURE CAPTIONS (Word Style "VA_Figure_Caption”).

FIGURE 1:

Numerical sample of silicalite-1 made of three repetitions in each direction of one period of the crystal.

FIGURE 2:

Example of equilibration run showing the amount of water adsorbed at $\mathrm{P} / \mathrm{P}_{0}=0.34$ from an initial configuration at $\mathrm{P} / \mathrm{P}_{0}=0.23$, as a function of the number of blocks of $10^{4}$ Monte-Carlo step.

\section{FIGURE 3:}

Water adsorption (circles) / desorption (triangles) isotherms in silicalite-1 as a function of relative pressure (first panel) or chemical potential (second panel).

FIGURE 4:

Water molecular configurations for four relative pressures $\left(\mathrm{P} / \mathrm{P}_{0}=0.0886,0.1477,0.2045,0.3409\right)$. The silicalite species are not represented.

\section{FIGURE 5:}

Configurational energy of water adsorbed in silicalite- 1 as a function of relative pressure or adsorbed amount. 
FIGURE 6:

Isosteric heat of adsorption of water in silicalite- 1 as a function of the adsorbed amount.

FIGURE 7:

Grand potential per unit volume of water adsorbed in silicalite as a function of the chemical potential. Circle: this work, dashed line: bulk water.

FIGURE 8:

Helmholtz free energy per water molecule adsorbed in silicalite as a function of the amount adsorbed.

FIGURE 9:

Entropy per water molecule adsorbed in silicalite as a function of the chemical potential.

FIGURE 10:

Water adsorption isotherm in silicalite-1 as a function of the chemical potential. Dashed lines and (+) signs: experimental data ${ }^{3,4,41-44}$. Circles: PN-TrAZ model (solid line is a guide to the eye). Triangles up : repulsive $b_{\text {Ow-Osilicalite }}$ reduced by $2.25 \%$. Triangles down : water partial charges reduced by $5 \%$. 
FIGURE 11:

Water dipole distribution calculated ab initio (see text) from (a) a 300K GCMC molecular configuration of $27 \mathrm{H}_{2} \mathrm{O}$ molecules confined in a silicalite unit cell (b) a bulk liquid configuration at room temperature $(1 \mathrm{~g} / \mathrm{cc})$. The vertical line shows the ab initio value of water dipole for a single molecule (gas phase). 
TABLES.

Table 1: PN-TrAZ parameters for water and silica species

\begin{tabular}{lcccc}
\hline \hline & \multicolumn{2}{c}{ water } & \multicolumn{2}{c}{ silicalite } \\
\cline { 2 - 5 } Atoms & $\mathrm{O}$ & $\mathrm{H}$ & $\mathrm{Si}$ & $\mathrm{O}$ \\
\hline $\mathrm{q}(\mathrm{e})$ & -0.82 & 0.41 & +2 & -1 \\
$\mathrm{~A}\left(E_{h}\right)$ & 247.7 & 1.338 & 6163.4 & 1543.5 \\
$\mathrm{~b}\left(a_{0}^{-1}\right)$ & 2.075 & 2.11 & 2.395 & 2.19 \\
Polarizabilities $\left(a_{0}{ }^{3}\right)$ & 7.56 & 2.655 & 2.36 & 8.03 \\
$\mathrm{~N}_{\mathrm{eff}}$ & 4.476 & 0.414 & 1.52 & 4.656 \\
\hline \hline
\end{tabular}

$a_{0}=0.529177 \AA, 1 E_{h}=3.157810^{5} \mathrm{~K}$

Table 2: Dispersion and repulsion parameters obtained in the framework of the PN-TrAZ model.

\begin{tabular}{ccccccc}
\hline \hline Water & Silicalite & & & & \\
species & species & $C_{6}\left(E_{h} a_{0}{ }^{6}\right)$ & $C_{8}\left(E_{h} a_{0}{ }^{8}\right)$ & $C_{10}\left(E_{h} a_{0}{ }^{10}\right)$ & $\mathrm{A}\left(E_{h}\right)$ & $\mathrm{b}\left(a_{0}{ }^{-1}\right)$ \\
\hline $\mathrm{H}$ & $\mathrm{O}$ & 8.3157 & 151.22 & 2668.6 & 45.444 & 2.149 \\
$\mathrm{H}$ & $\mathrm{Si}$ & 2.4874 & 32.919 & - & 90.811 & 2.243 \\
$\mathrm{O}$ & $\mathrm{O}$ & 34.850 & 735.319 & 13540.4 & 618.3 & 2.131 \\
$\mathrm{O}$ & $\mathrm{Si}$ & 10.513 & 172.72 & - & 1235.5 & 2.223 \\
\hline \hline
\end{tabular}


Figure 1

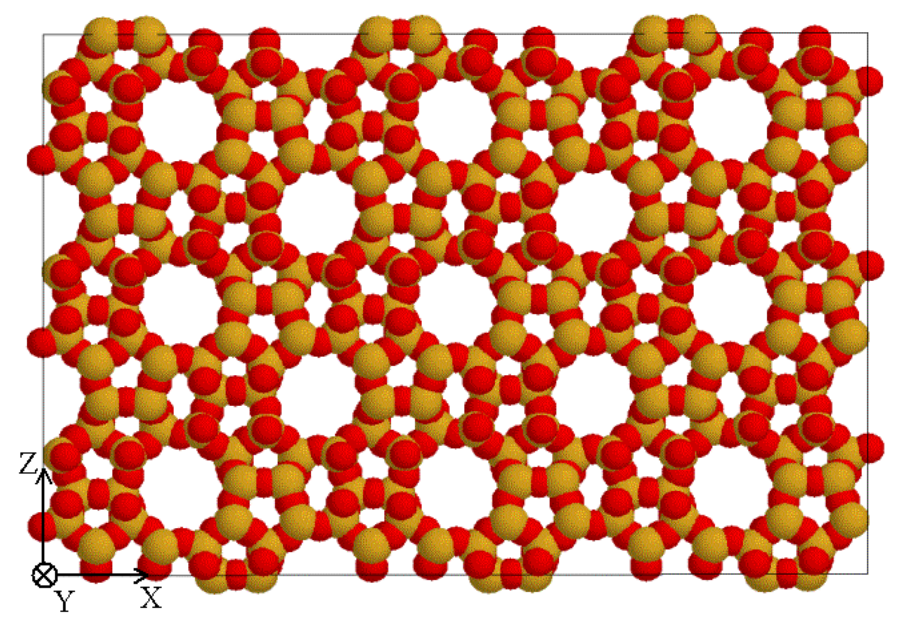


Figure 2

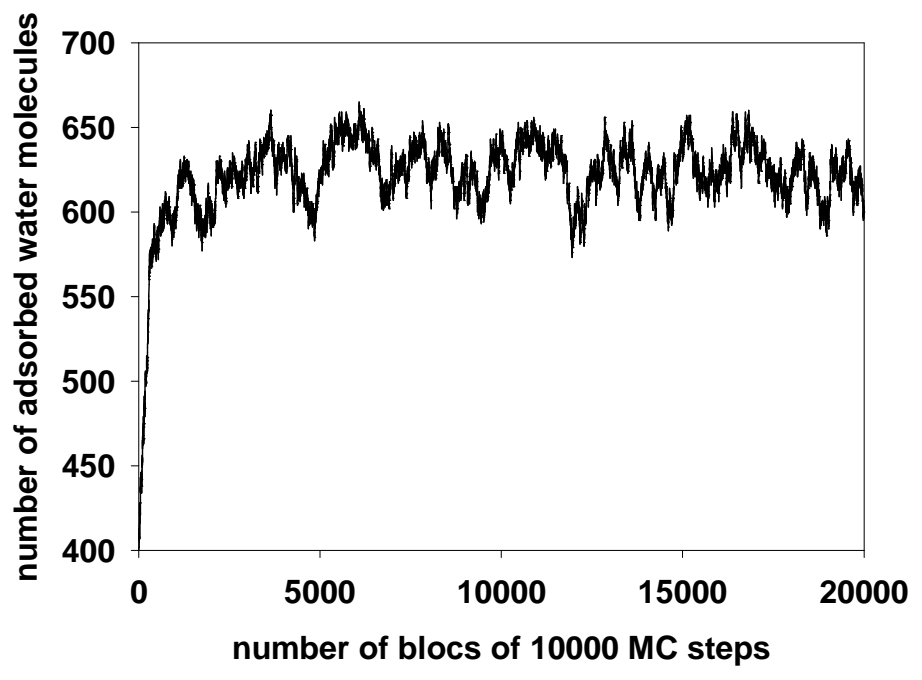


Figure 3
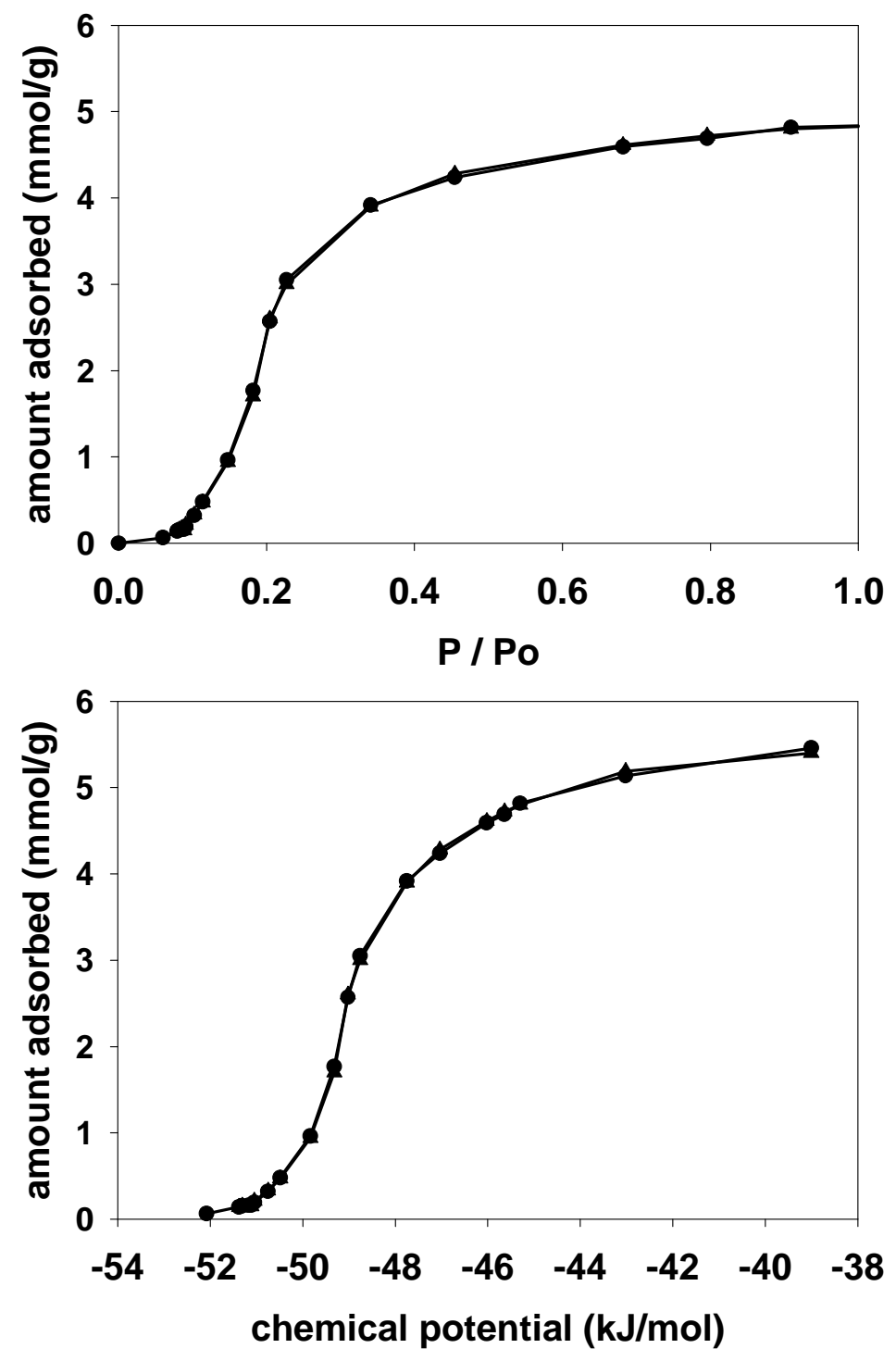
Figure 4
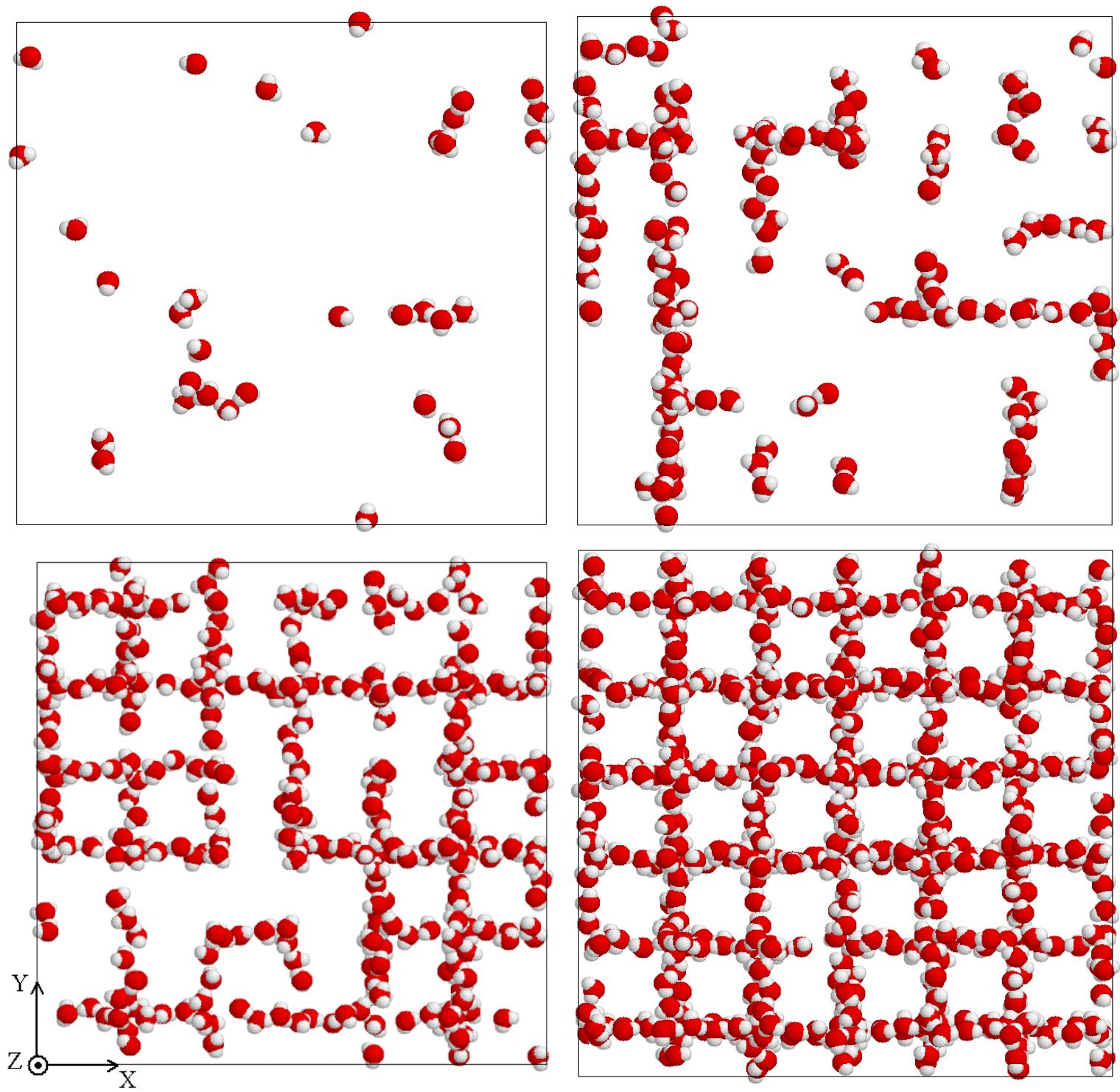
Figure 5
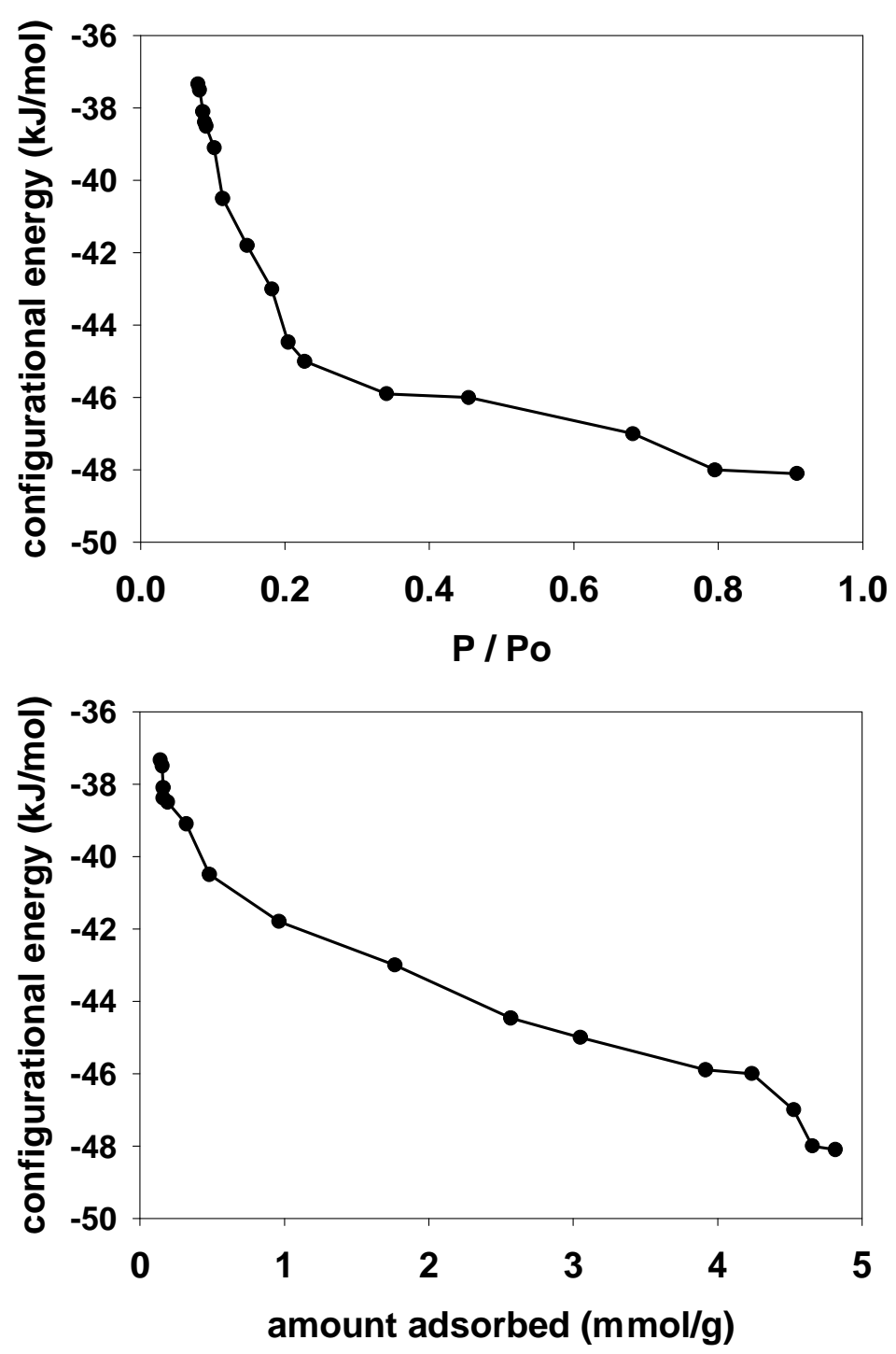
Figure 6

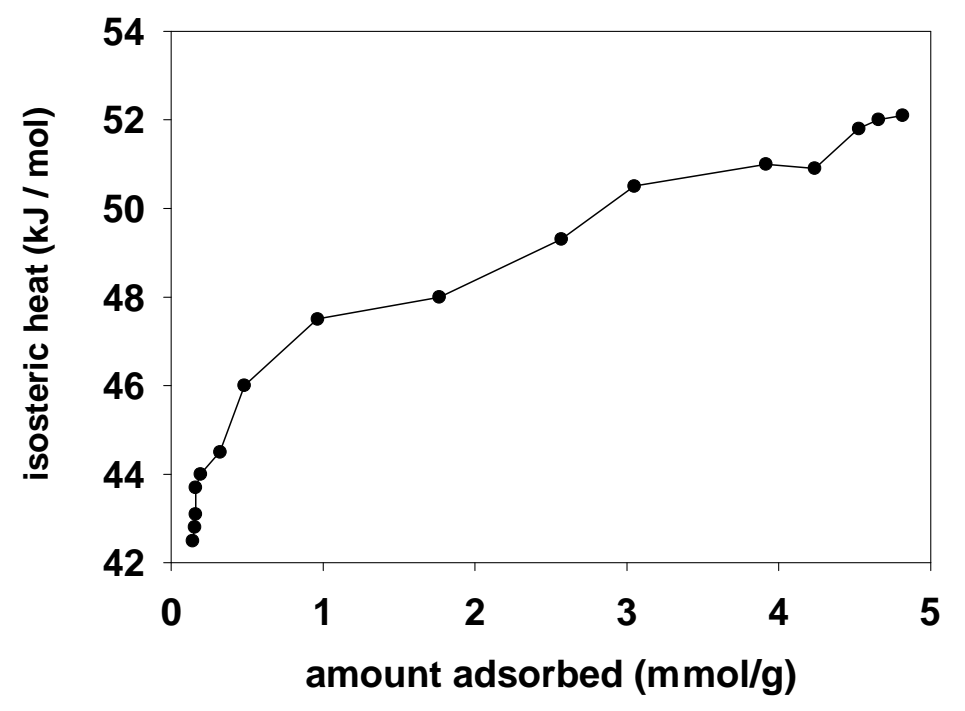


Figure 7

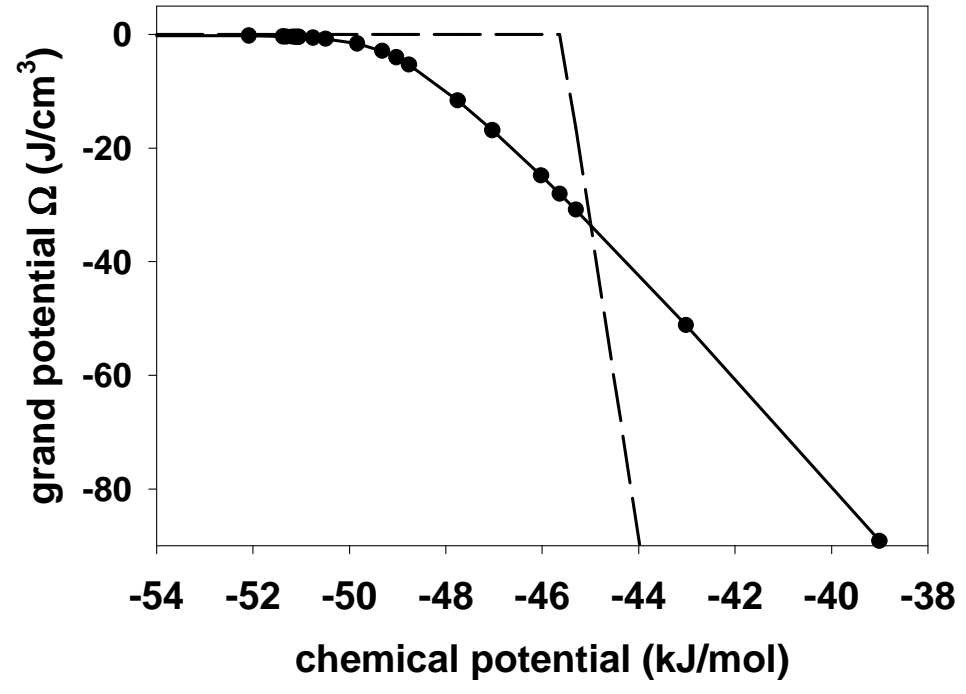


Figure 8

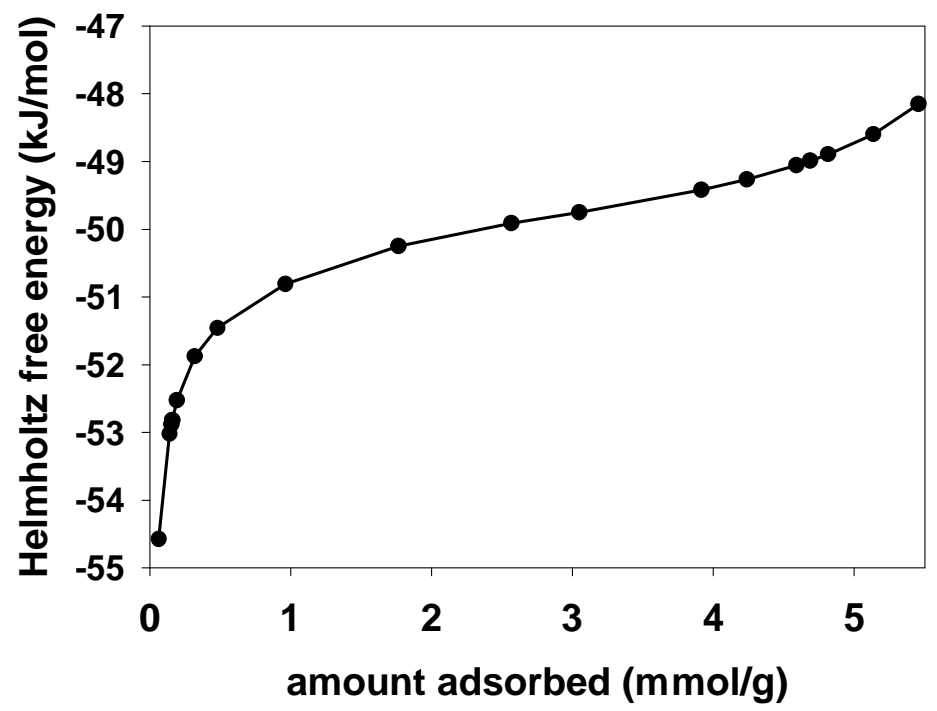


Figure 9

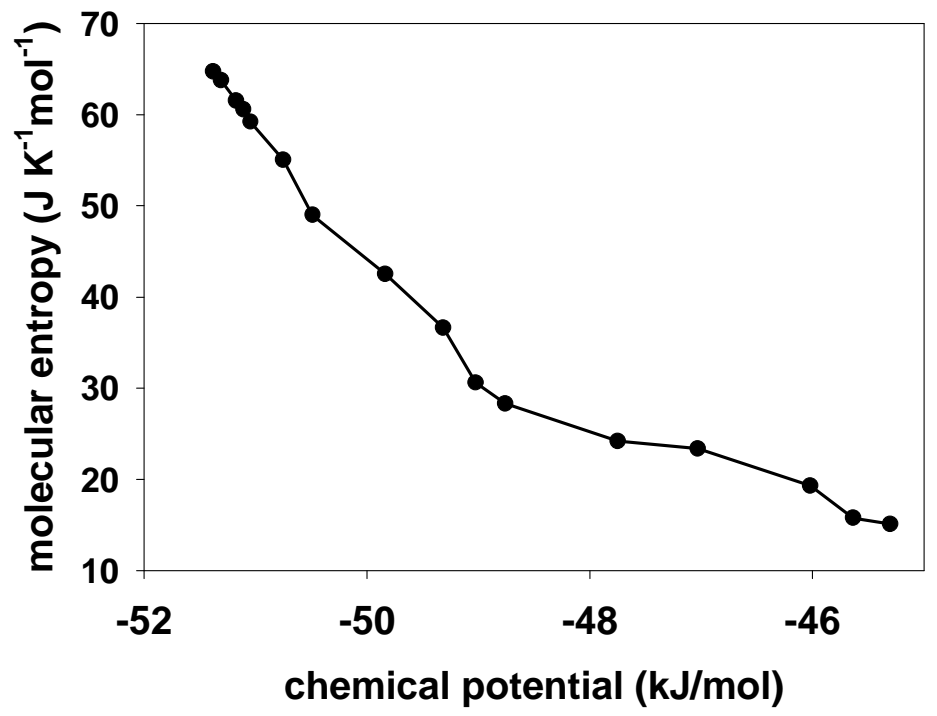


Figure 10

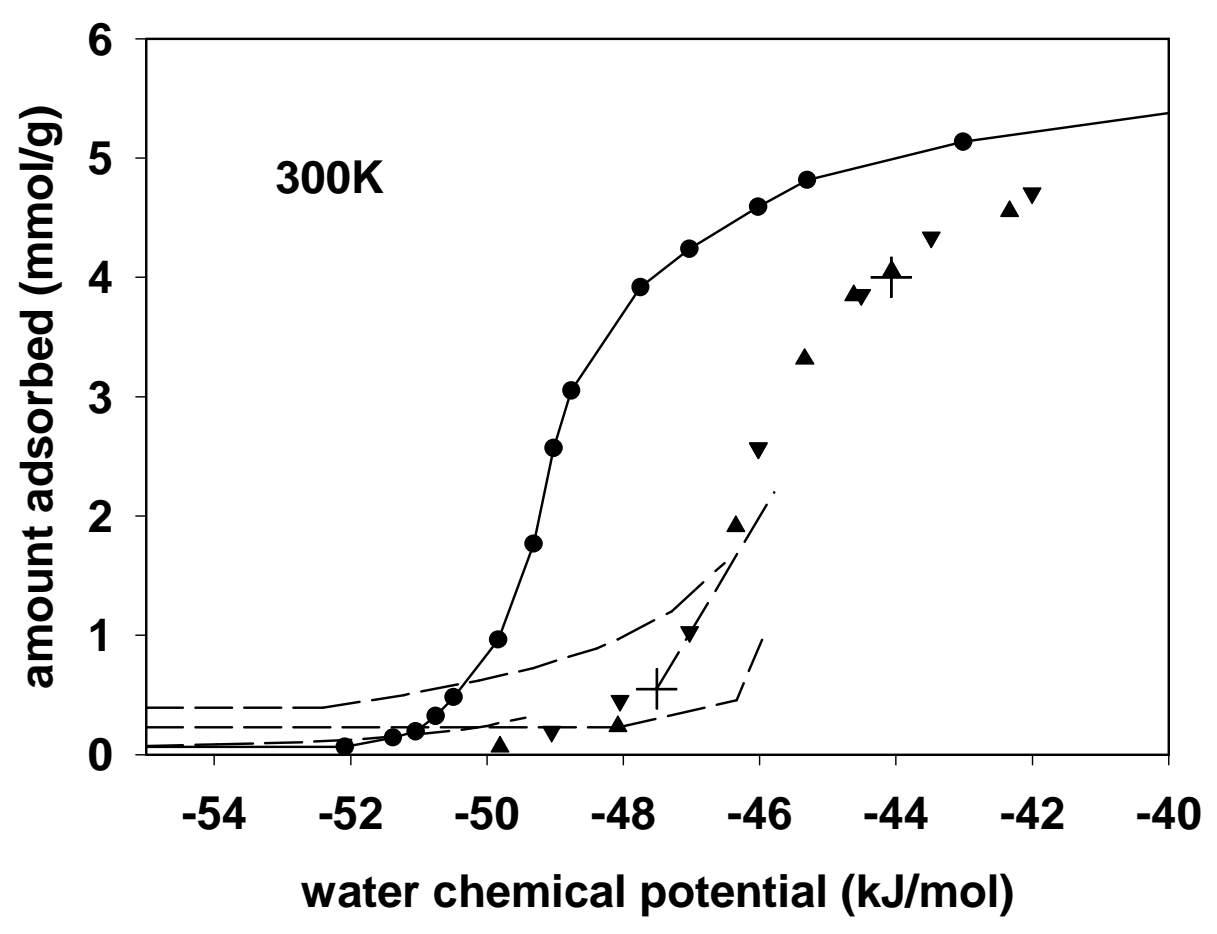


Figure 11

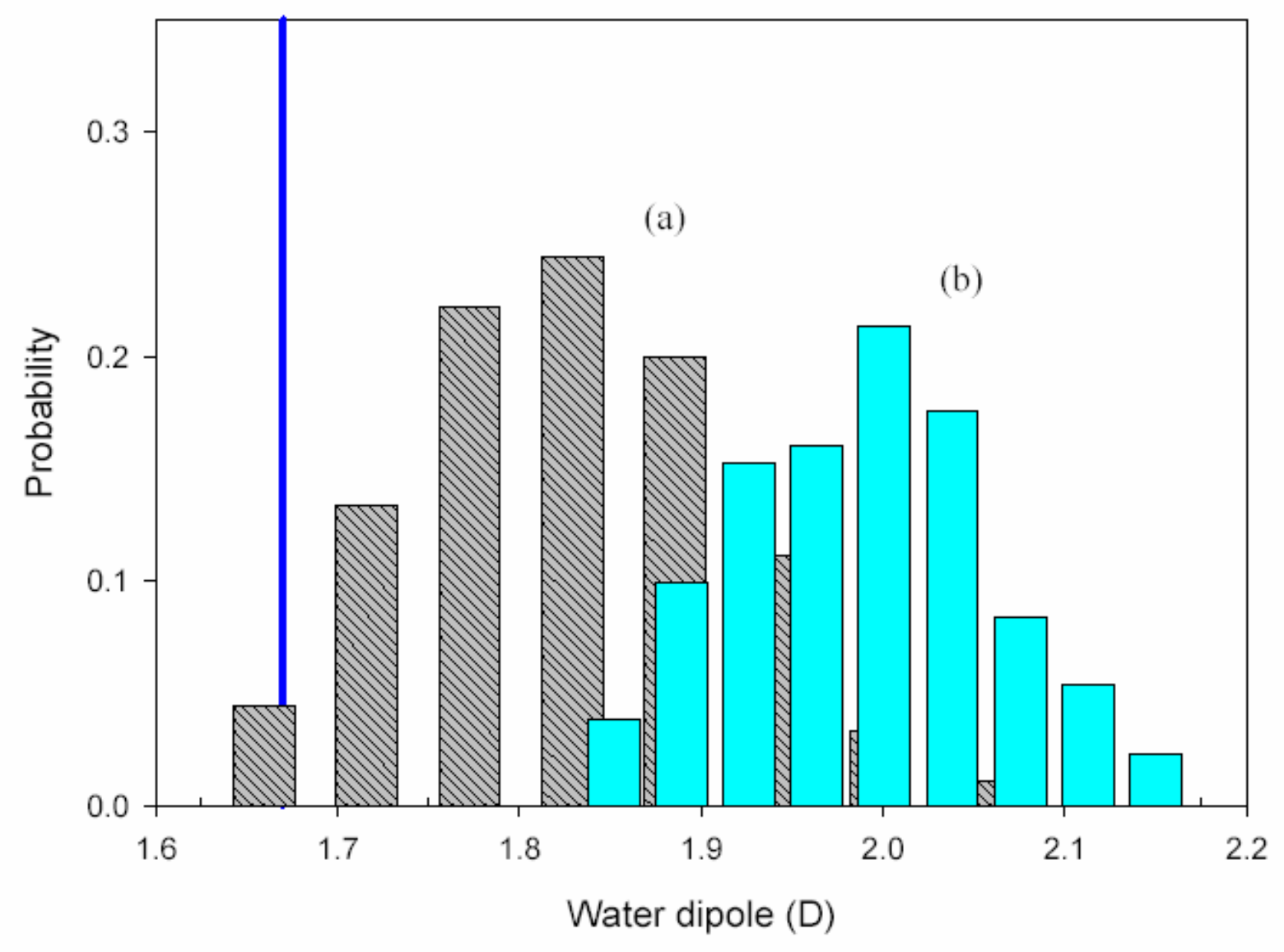

